\title{
TRP Channels in Agonist-Induced Proliferation of Prostate Cancer Cells
}

\author{
Y. M. Shuba ${ }^{a}$, R. Skryma ${ }^{b}$, and N. Prevarskaya ${ }^{b}$ \\ a International Center of Molecular Physiology NASU, Kiev, Ukraine; \\ e-mail: yshuba@biph.kiev.ua \\ ${ }^{b}$ Laboratoire de Physiologie Cellulaire, Universite des Sciences et Technologies de Lille, Villeneuve d'Ascq, France \\ DOI: $10.1134 / \mathrm{S} 1990747809030453$
}

Agonist-induced stimulation of $\alpha 1$-adrenoreceptors $(\alpha 1-\mathrm{AR})$ of the cultured primary human prostate cancer (PCa) epithelial cells results in the enhancement of proliferative activity accompanied by the oscillations of the intracellular calcium concentration $\left(\left[\mathrm{Ca}^{2+}\right]_{i}\right)$, increase of store-independent calcium entry, and activation of the transcription factor NFAT (nuclear factor of activated $\mathrm{T}$ cells). Calcium entry in response to the $\alpha 1$-ARs stimulation is primarily mediated by TRPC6 (transient receptor potential canonical 6) cationic channel: antisense TRPC6 depletion decreases calcium entry, suppresses $\left[\mathrm{Ca}^{2+}\right]_{i}$ oscillations and slows down proliferation caused by the $\alpha 1-\mathrm{AR}$ agonist phenylephrine. On the contrary, stimulation of purinergic P2Y receptors (P2Y-R) leads to complete cessation of proliferative activity of human $\mathrm{PCa}$ cells due to enhancement of sustained store-dependent calcium entry, which is mainly mediated by TRPC 1 and TRPC4 channels without NFAT involvement. Expression and channel-like function of another member of TRP channels family, cold/menthol-sensitive TRPM8 (melastatin 8), was dependent on the presence of functional androgen receptor and was characteristic of only apical phenotype of PCa epithelial cells. Our results establish TRPC6 and TRPC1/4 channels as important determinants in the control of PCa epithelial cells proliferation and TRPM8 channel as a marker of the PCa stages and androgen dependency.

The work was in part supported by INTAS (project no. 05-1000008-8223). 